\title{
SUPPORTS AND SINGULAR SUPPORTS OF PSEUDOMEASURES
}

\author{
R. E. EDWARDS
}

(Received 31 December 1964)

\section{Summary}

Let $G$ denote a Hausdorff locally compact Abelian group which is nondiscrete and second countable. The main results (Theorems (2.2) and (2.3)) assert that, for any closed subset $E$ of $G$ there exists a pseudomeasure $s$ on $G$ whose singular support is $E$; and that if no portion of $E$ is a Helson set, then such an $s$ may be chosen having its support equal to $E$. There follow (Corollaries (2.2.4) and (2.3.2)) sufficient conditions for the relations

$$
\operatorname{supp} s=F, \quad \operatorname{sing} \operatorname{supp} s=E
$$

to hold for some pseudomeasure $s, E$ and $F$ being given closed subsets of $G$. These results are analogues and refinements of a theorem of Pollard [4] for the case $G=R$, which asserts the existence of a function in $L^{\infty}(R)$ whose spectrum coincides with any preassigned closed subset of $R$.

The study of supports and singular supports of pseudomeasures is in part prompted by the aid afforded by these concepts in handling convolution equations of the type $\mu * f=g$ with $\mu \in M(G)$ and $f, g \in L^{\infty}(G)$, and of problems concerning spans of translates of bounded functions; see Remark (2.2.5).

\section{Preliminaries}

Throughout this note, $G$ denotes a Hausdorff locally compact Abelian group and $X$ its character group. Pseudomeasures on $G$ are here defined as continuous linear functionals on the space $A(G)$ of functions $u$ on $G$ of the form

$$
u(x)=\int_{x} v(\xi) \xi(x) d \xi,
$$

where $d \xi$ denotes Haar measure on $X$ and $v \in L^{1}(X)$, the norm on $A(G)$ being given by

$$
\|u\|_{A}=\|v\|_{1} \equiv \int_{X}|v(\xi)| \mathrm{d} \xi
$$


cf. Appendices II, III of [1] and [2], pp. 8-9. We denote by $P(G)$ the space of pseudomeasures on $G$. It is known, and quite easy to verify, that the Fourier transformation can be defined for functions in $L^{\infty}(X)$ in such a way as to realise a linear isometry of $L^{\infty}(X)$ onto $P(G)$.

There is a natural injection of $M(G)$, the space of bounded Radon measures on $G$, into $P(G)$ (though in general $P(G)$ will embrace also certain unbounded Radon measures on $G$ ).

Pseudomeasures can be studied locally: if $U$ is an open subset of $G$, and if $s$ and $s^{\prime}$ are pseudomeasures on $G$, we write

$$
s=s^{\prime} \text { on } U
$$

to signify that $s(u)=s^{\prime}(u)$ for each $u \in A(G)$ whose support supp $u$ is contained in $U$. (It is equivalent to demand that $s(u)=s^{\prime}(u)$ for each $u \in A(G)$ such that supp $u$ is compact and contained in $U$ ).

If $s \in P(G)$, the support [resp. singular support] of $s$, denoted by supp $s$ [resp. sing supp $s$ ], is defined to be the complement, relative to $G$, of the open set of all points $x \in G$ on some open neighbourhood of which $s$ is equal to zero [resp. to a measure]. This both supp $s$ and sing supp $s$ are closed subsets of $G$, and sing supp $s$ supp $s$. Notice that if $s$ is equal on some open set $U$ to a Radon measure, then, for any relatively compact open set $V C U$, one has $s=\mu$ on $V$ for some $\mu \in M(G)$.

Given a closed set $E \subset G$, the relation supp $s \subset E$ signifies that

$$
s(u)=0
$$

for each $u \in A(G)$ for which supp $u \subset E^{\prime}$ (the complement of $E$ relative to $G$ ), or, what is equivalent, for each $u \in A(G)$ for which supp $u$ is a compact subset of $E^{\prime}$. A generally stronger condition on $s$ is to demand that (1.1) holds for each $u \in A(G)$ which vanishes on $E$. We shall write

$$
\begin{aligned}
P_{E}(G)= & \{s \in P(G): \operatorname{supp} s \subset E\}, \\
P_{E}^{0}(G)= & \{s \in P(G): s(u)=0 \text { for all } u \in A(G) \\
& \text { satisfying } u(E)=0\} \subset P_{E}(G) .
\end{aligned}
$$

It follows from known results that $P_{E}(G)=P_{E}^{0}(G)$ if and only if $E$ is a spectral synthesis set (see [2], pp. 158-161; we are here regarding $G$ as the character group of $X$ ), but we shall make no use of this fact.

\section{The main results}

Our proof of the existence of a pseudomeasure having as singular support a given closed set depends on the construction of such a pseudomeasure for the special case in which the closed set is reduced to a point, say the zero element of $G$. Since the latter problem is handled in a relatively constructive 
manner, we deal with it separately in Theorem (2.1). The general result appears in Theorem (2.2) and is existential in nature, as also is Theorem (2.3).

(2.1) Theorem. Suppose that $G$ is nondiscrete and first countable. Let $N_{0}$ be any neighbourhood of 0 in $G$. There exists on $G$ a function $f$ with the following properties:

(i) $f$ is continuous (and even $C^{\infty}$ if $G$ is a Lie group) on $W=G \mid\{0\}$;

(ii) $f=0$ on $N_{0}^{\prime}$;

(iii) $\int_{N^{\prime}}|f| d x<\infty$ for any neighbourhood $N$ of 0 in $G$, and $\int_{G}|f| d x=\infty$;

(iv) there is a decreasing base $\left(U_{n}\right)_{n=1}^{\infty}$ of neighbourhoods of 0 in $G$ such that the formula

$$
s_{0}(u)=\lim _{n \rightarrow \infty} \int_{U_{n}^{\prime}} f(x) u(x) d x \quad(u \in A(G))
$$

defines a pseudomeasure $s_{0} \in P(G)$ for which sing supp $s_{0}=\{0\}$. [In distributional language, $s_{0}$ is thus the Cauchy principal value of the function $f$.]

$(v)$ the Fourier transform $\hat{s}_{0} \in C_{0}(X)$ [the space of continuous functions which vanish at infinity on the noncompact character group $X$ of $G]$.

Proof. Since $G$ is nondiscrete and first countable, we may choose a decreasing base $\left(U_{n}\right)_{n=1}^{\infty}$ of open neighbourhoods of 0 in $G$ such that $\bar{U}_{1} \subset N_{0}$ and $V_{n}=U_{n} \mid \vec{U}_{n+1}$ is nonvoid. Let $K_{n}$ be a compact subset of $V_{n}$ having interior points. Then ([2], Theorem 5.6.10) $K_{n}$ is not a Helson set and so ([2], Theorem 5.6.3) one can find a measure $\alpha_{n} \in M(G)$ such that supp $\alpha_{n} \subset K_{n}$ and

$$
\int_{G} d\left|\alpha_{n}\right| \geqq n, \quad|| \hat{\alpha}_{n} \|_{\infty} \leqq 1 / n^{2} .
$$

By regularisation, one may assume that $\mathrm{d} \alpha_{n}=f_{n} \mathrm{~d} x$, where $f_{n}$ is a continuous function [even a $C^{\infty}$ function, if $G$ is a Lie group] with a compact support contained in $K_{n}$. The series $\sum_{n=1}^{\infty} \alpha_{n}$ is then convergent in $P(G)$. Denote its sum by $s_{0}$. Then $(v)$ is true, thanks to the Riemann-Lebesgue lemma and the uniform convergence of the series $\sum \hat{\alpha}_{n}$. At the same time, the series $\sum_{n=1}^{\infty} f_{n}(x)$ is convergent for each $x \in G$, and it is easy to see that the sum-function $f$ has properties (i), (ii) and (iii). In addition, if $u \in A(G)$ we have

$$
\begin{aligned}
s_{0}(u) & =\lim _{n} \sum_{m=1}^{n} \int_{G} u d \alpha_{m}=\lim _{n} \int_{G}\left(\sum_{m=1}^{n} f_{m}\right) u d x \\
& =\lim _{n} \int_{U_{m+1}^{\prime}} f u d x,
\end{aligned}
$$

so that (1) is established. It remains to show that sing supp $s_{0}=\{0\}$.

Now if $N$ is any compact neighbourhood of 0 , (iii) combines with the weak relative compactness of norm-bounded subsets of $M\left(N^{\prime}\right)$ to show that 
$s_{0}$ is equal on $N^{\prime}$ to a measure $\beta_{N} \in M(G)$. This shows at once that sing supp $s_{0} \subset N$ for any compact neighbourhood $N$ of 0 , and so that $\operatorname{sing} \operatorname{supp} s_{0} \subset\{0\}$. On the other hand, if 0 did not belong to sing supp $s_{0}$, there would exist an open neighbourhood $U$ of 0 and a measure $\lambda \in M(G)$ such that $s_{0}=\lambda$ on $U$. Then, taking $N \subset U$, we should have $s_{0}=\lambda$ on $U, s_{0}=\beta_{N}$ on $N^{\prime}$, the open sets $U$ and $N^{\prime}$ covering $G$. At this point Lemma (2.1.1) would apply to show that $s_{0}$ would be equal globally to a measure $\mu \in M(G)$. This $\mu$ would necessarily coincide on $V_{n}$ with $\alpha_{n}$, so that

$$
\int_{G} d|\mu| \geqq \int_{V_{n}} d|\mu|=\int_{V_{n}} d\left|\alpha_{n}\right|=\int_{G} d\left|\alpha_{n}\right| \geqq n,
$$

and this for all $n$. This absurdity shows that $0 \in \operatorname{sing}$ supp $s_{0}$ and so completes the proof.

(2.1.1) Lemma. Suppose that $A$ and $B$ are open subsets of $G$, that $s \in P(G)$, that $\alpha$ and $\beta$ belong to $M(G)$, and that $s=\alpha$ on $A$ and $s=\beta$ on $B$. Then $s$ is equal on $A \cup B$ to an element of $M(G)$.

Proof. It suffices to show that

$$
|s(u)| \leqq \text { const. }\|u\|_{\infty}
$$

for each $u \in A(G)$ having a compact support $K \subset A \cup B$.

Now it is easily established that positive functions $a, b \in A(G)$ may be chosen so that supp $a \subset A$, supp $b \subset B$ and $a+b=1$ on $K$. Then

$$
s(u)=s(a u)+s(b u) \text {. }
$$

But supp au $\subset A$ and so

$$
\begin{aligned}
\mid s(a u \mid & =\int_{G} a u d \alpha \leqq \int_{G} d|\alpha| \cdot\|a u\|_{\infty} \\
& \leqq \int_{G} d|x| \cdot\|u\|_{\infty} .
\end{aligned}
$$

Similarly,

$$
|s(b u)| \leqq \int_{G} d|\beta| \cdot\|u\|_{\infty}
$$

These inequalities combine with (3) to yield (2), and the lemma is established.

(2.1.2) Remark. If the formula (1) is to yield a pseudomeasure $s_{0}$ with a singularity at 0 , the second clause of (2.1.iii) is necessarily true. This ..sules from the following remark, which is an analogue of Riemann's theorem about the removability of isolated singularities of bounded analytic functions:

If $s \in P(G)$ is such that, for some neighbourhood $U$ of $0, s$ is equal on $U \backslash\{0\}$ to a bounded measure, then $s$ is equal on $U$ itself to a bounded measure. For the hypothesis ensures that for some number $c>0$ one has 


$$
|s(u)| \leqq c .\|u\|_{\infty}
$$

whenever $u \in A(G)$ and supp $u \subset U \backslash\{0\}$. From a known result ([2], Theorem 2.6.4) it follows first that the same inequality holds for any $u \in A(G)$ for which supp $u \subset U$ and $u(0)=0$, and then by a simple argument that

$$
|s(u)| \leqq 2 c \cdot\|u\|_{\infty}
$$

for any $u \in A(G)$ satisfying supp $u \subset U$. From this the stated conclusion follows.

The same arguments show that if $E$ is a $C$-set in $G([2], \S 7.5 .1), U$ a a neighbourhood of $E$, and if $s \in P(G)$ is equal on $U \cap E^{\prime}$ to a bounded measure, then there exists a measure $\mu \in M(G)$ such that

$$
s(u)=\int_{G} u d \mu
$$

for each $u \in A(G)$ such that supp $u \subset U$ and $u(E)=0$.

In what follows we denote by $M_{E}(G)$ the set of measures $\mu \in M(G)$ satisfying supp $\mu \subset E$, by $M_{B}^{+}(G)$ the set of positive elements of $M_{E}(G)$, and by $L_{E}^{1}(G)$ the set of elements of $L^{1}(G)$ defined by integrable functions which vanish on $E^{\prime}$. Besides this, given a finite or infinite sequence $\left(a_{n}\right)$ of points of $G$, we shall write $D$ for the set of purely discrete measures $\mu \in M(G)$ of the form

$$
\mu=\sum c_{n} \varepsilon_{a_{n}},
$$

where $\varepsilon_{a}$ denotes the Dirac measure placed at $a$, and where the $c_{n}$ are nonnegative numbers satisfying $\sum c_{n}<\infty$. It is easily verified that $D$ is a closed convex cone in $M(G)$. If $E$ is a closed subset of $G$, and if each $a_{n}$ belongs to $E$, then $D \subset M_{B}^{+}(G)$.

(2.2) THEOREM. Suppose that $G$ is nondiscrete and first countable. Let $E$ be a closed subset of $G$ satisfying the following condition:

(C) There exists a sequence $\left(U_{n}\right)_{n=1}^{\infty}$ of open subsets of $G$ which meet $E$ and are such that any open subset of $G$ meeting $E$ contains $U_{n}$ for some $n$ (i.e., $E$ is a second countable subspace of $G$ ).

Let $s_{0}$ be as in Theorem (2.1) and suppose that the points $a_{n}(n=1,2, \ldots)$ form a countable dense subset of $E$. Then:

(i) There exists numbers $c_{n} \geqq 0$ such that $\sum c_{n}<\infty$ and such that the measure $\mu=\sum c_{n} \varepsilon_{\alpha_{n}}$ satisfies

$$
\text { sing supp } s_{0} * \mu=E \text {. }
$$

(ii) If further $E \cap U$ has strictly positive Haar measure whenever $U$ is an open subset of $G$ meeting $E$, there is a positive function $f \in L_{E}^{1}(G)$ such that

$$
\text { sing supp } s_{0} * f=E \text {. }
$$


Proof. (i) As will be shown in Lemma (2.2.1) infra, sing supp $s_{0} * \mu$ $C E$ for any $\mu \in M_{E}(G)$. Thus, using the notation introduced immediately prior to the statement of the theorem, it suffices to show that a measure $\mu \in D$ exists for which sing supp $s_{0} * \mu \supset E$. We will in fact show that the negation of this leads to a contradiction.

Putting $D_{n, k}(n, k=1,2, \cdots)$ for the set of $\mu \in D$ such that $s_{0} * \mu=\lambda$ on $U_{n}$ for some $\lambda \in M(G)$ satisfying $\|\lambda\|_{M} \leqq k$, the said negation would combine with (C) (and the evident remark that one may in (C) assume each $U_{n}$ to be relatively compact) to entail that

$$
D=\bigcup\left\{D_{n, k}: n, k=1,2, \ldots\right\} .
$$

In any case, the weak relative compactness of norm-bounded subsets of $M(G)$ goes to show that each $D_{n, k}$ is closed in $D$. Since also $D$ is a convex cone, the category theorem and (4) would combine to show that some $D_{n, k}$ is a neighbourhood of zero in $D$. In other words, there is a number $c>0$ such that each $\mu \in D$ satisfies

$$
s_{0} * \mu=\lambda \text { on } U_{n},
$$

where $\lambda=\lambda(\mu) \in M(G)$ satisfies

$$
\|\lambda\|_{M} \leqq c \cdot\|\mu\|_{M} .
$$

Taking $a_{i} \in U_{n} \cap E$ and $\mu=\varepsilon_{a_{i}}$, we see that $s_{0} * \varepsilon_{a_{t}}$ is a measure on $U_{n}$. But then $s_{0}$ itself would be a measure on $U_{n}-a_{i}$. Since $U_{n}-a_{i}$ is a neighbourhood of 0 , this contradicts (iv) of Theorem (2.1), and (i) is thereby established.

(ii) The same type of argument leads to (5) and (6) once more, the sole difference being that $\mu$ is to be replaced by a positive function $f \in L_{E}^{1}(G)$. Owing to the final hypothesis on $E$, however we can now take a norm-bounded sequence $\left(f_{r}\right)$ or positive elements of $L_{E}^{1}(G)$ which converges weakly to $\varepsilon_{a_{i}}$. By $(6)$ the corresponding measures $\lambda_{r}$ may be chosen to have bounded norms, and so to admit a weak limiting point $\lambda \in M(G)$. It would then appear that again $s_{0} * \varepsilon_{a_{i}}$ is equal on $U_{n}$ to a measure, from which point the proof proceeds as before.

(2.2.1) Lemma. Let $s_{0}$ be as in Theorem (2.1). Then sing supp $s_{0} * \mu \subset E$ whenever $\mu \in M_{E}(G)$.

Proof. We use the fact that $s_{0} * \mu$ is representable as the vectorvalued integral

$$
s_{0} * \mu=\int_{E}\left(s_{0} * \varepsilon_{a}\right) d \mu(a) .
$$

Choose any $x_{0} \in E^{\prime}$ and any compact symmetric neighbourhood $N$ of 0 
such that $x_{0}+N$ does not meet $E$. Then, if $a \in E, a+N$ does not contain $x_{0}$. An open neighbourhood $U$ of $x_{0}$ may then be found so that $(a+N) \cap$ $U=\emptyset$ for all $a \in E$. The union $V$ of the sets $U-a(a \in E)$ is then open and contained in $N^{\prime}$. Hence as was seen in the proof of Theorem (2.1), $s_{0}$ is equal on $V$ to a measure $\beta \in M(G)$. A fortiori, $s_{0}=\beta$ on $U-a$ for all $a \in E$, and $s_{0} * \varepsilon_{a}=\beta * \varepsilon_{a}$ on $U$ for all $a \in E$. Reference to (7) shows that

$$
s_{0} * \mu=\int_{E}\left(\beta * \varepsilon_{a}\right) d \mu(a) \text { on } U .
$$

The integral on the right here obviously represents an element of $M(G)$, whence it is plain that $x_{0}$ does not belong to sing supp $s_{0} * \mu$. The proof is complete.

Remark. Similar arguments show that, if $s \in P(G)$ and $\mu \in M(G)$, then the relation

$$
\text { sing supp } s * \mu \subset \text { sing supp } s+\operatorname{supp} \mu
$$

holds provided at least one summand on the right is compact.

(2.2.2) Remark. According to (v) of Theorem (2.1), the pseudomeasure $s=s_{0} * \mu$ referred to in Theorem (2.2) is such that $s \in C_{0}(X)$.

(2.2.3) Remark. Arguments similar to those used in the proof of Theorem (2.2) will show that, if $G$ is second countable and nondiscrete, and if $s \in P(G)$ is such that sing supp $s \neq \emptyset$, then there exists an $f \in L^{1}(G)$ such that sing $\operatorname{supp} s * f=G$.

(2.2.4) Corollary. Suppose that $G$ is nondiscrete and second countable, that $E \subset F$ are closed subset of $G$, and that $F$ is a neighbourhood of $E$. Then there exists an $s \in P(G)$ such that

$$
\text { supp } s=F, \quad \text { sing supp } s=E .
$$

PRoof. It is easily seen that there exists a measure $\lambda \in M(G)$ satisfying supp $\lambda=F$. By Theorem (2.2), there exists $s_{1} \in P(G)$ satisfying sing supp $s_{1}=E$. Since $F$ is a neighbourhood of $E$, we may obviously assume that supp $s_{1} \subset F$. (If this is not already the case, it suffices to multiply $s_{1}$ by an element $u$ of $A(G)$ which is nonvanishing on $E$ and satisfies supp $u \subset F$.) The pseudomeasure $s=s_{1}+\lambda$ then satisfies all our requirements.

(2.2.5) Remarks. (i) Suppose that $G$ is noncompact and second countable. By interchanging $G$ and $X$, Theorem (2.2) shows that there exists $f \in C_{0}(G)$ such that sing supp $\hat{f}=X$. Such an $f$ is characterised by the property that $\lambda=0$ is the only element of $M(G)$ such that $\lambda * f$ is the FourierStieltjes transform of an element of $M(X)$. If $G=R^{n}$, one can even choose such an $f$ which is analytic and such that it and each of its partial derivatives belong to $C_{0}\left(R^{n}\right)$. 
As a consequence the function $f$ possesses the following remarkable translational properties: if $g$ is the Fourier-Stieltjes transform of an element of $M(X)$, then the span of translates of $f+g$ is strictly dense in the space of all bounded continuous functions on $G$; and if further $g \in C_{0}(G)$, the said span is unformly dense in $C_{0}(G)$. (Both assertions follow from a direct application of the Hahn-Banach theorem; the strict topology is that defined by the seminorms $N_{k}(h)=\|k h\|_{\infty}$ when $k$ ranges over $C_{0}(G)$. The dual of $C_{0}(G)$ relative to the uniform topology, and that of the space of bounded continuous functions relative to the strict topology, is identifiable with $M(G)$. See [7].)

(ii) Suppose that $G$ is nondiscrete and second countable and that $X$ contains at least one infinite discrete subgroup. Then it is shown in Theorem 2.5 of [5] that there exists a pseudomeasure $s_{1} \in P(G)$ such that sing supp $s_{1}=G$ and such that $s_{1}$ is the inverse Fourier transform of a function in $L^{p}(X)$ for all $p$ satisfying $2<p \leqq \infty$. On the other hand, if $U$ is any open subset of $G$, there exists a function $f \in L^{1}(X)$ such that $U$ is the nonzero set of the inverse Fourier transform $F$ of $f$ (see the remark preceding Theorem (6.2) of [6]). We can moreover arrange that $f$ is bounded, and so belongs to $L^{q}(X)$ for every $q$ satisfying $1 \leqq q \leqq \infty$. Then $s=F \cdot s_{1} \in P(G)$, sing supp $s=\bar{U}$, and $s$ is the inverse Fourier transform of a function belonging to $C_{0}(X)$ and to $L^{p}(X)$ for every $p>2$. As before, if $G=R^{n}$, we may arrange that this latter function is even analytic and has partial derivatives in $C_{0}\left(R^{n}\right)$.

This result constitutes a variant of Theorem (2.2) for the case in which $E$ is the closure of an open subset of $G$.

We turn next to considering when a pseudomeasure $s$ exists satisfying $\operatorname{supp} s=\operatorname{sing} \operatorname{supp} s=E$ for a given closed subset $E$ of $G$.

(2.3) Theorem. Let $E$ be a closed subset of $G$ which satisfies $(C)$ of Theorem (2.2) and also the following condition.

$(N H)$ For any relatively compact open subset $U$ of $G$ which meets $E, E \cap \bar{U}$ is not a Helson set ([2], p. 114).

Then there exists an $s \in P_{E}^{0}(G)\left[C P_{E}(G)\right]$ such that $\operatorname{sing} \operatorname{supp} s=E$.

Proof. This proceeds by contradiction, starting from the assumption that the assertion of the theorem is false.

On the basis of this last assumption it follows that to each $s \in P_{E}^{0}(G)$ corresponds a point $x \in E$ and a neighbourhood $U$ of $x$ on which $s$ is a measure. According to (C), there exists an $n$ such that $s$ is equal on $U_{n}$ to a measure in $M(G)$.

For $n, k=1,2, \cdots$, denote by $P_{n, k}$ the set of $s \in P_{E}^{0}(G)$ such that $s=\mu$ on $U_{n}$, where $\mu \in M(G)$ and $\|\mu\|_{M} \leqq k$. (We are here using $\|\cdot\|_{M}$ to denote the usual norm on $M(G)$.) Now $P_{E}^{0}(G)$ is easily seen to be a closed subspace of the Banach space $P(G)$, the norm on the latter space being that dual to 
the norm on $A(G)$, and denoted hereafter by $\|\cdot\|_{p}$. Moreover, $P_{n, k}$ is closed in $P(G)$, as follows from the weak relative compactness of norm-bounded subsets of $M(G)$. Since, as we have seen, $P_{E}^{0}(G)$ is the union of the $P_{n, k}$, Baire's category theorem affirms the existence of a pair $(n, k)$ such that $P_{n, k}$ contains some nonvoid relatively open subset of $P_{E}^{0}(G)$. This signifies that, for some $n$ and some number $c \geqq 0$, to each $s \in P_{E}^{0}(G)$ corresponds a measure $\mu \in M(G)$ such that

$$
s=\mu \text { on } U_{n}, \quad\|\mu\|_{M} \leqq c .\|s\|_{P} .
$$

Take now a relatively compact open set $U$ meeting $E$ and such that $\bar{U} \subset U_{n}$, and then a function $u \in A(G)$ such that $u=1$ on $U$ and supp $u \subset U_{n}$. Then, if $H=\bar{U} \cap E$, and if $s \in P_{H}^{0}(G)$, (8) entails that $s=u \cdot s=u \cdot \mu$ globally. Thus each $s \in P_{H}^{0}(G)$ is globally equal to a measure in $M(G)$. But, by Lemma (3.1) infra this implies that $H$ is a Helson set, contrary to $(N H)$. This contradiction completes the proof.

(2.3.1) Remark. Condition $(\mathrm{NH})$ is essential for the validity of Theorem (2.3). Indeed, if $U$ is a relatively compact open subset of $G$ which meets $E$ and which is such that $\bar{U} \cap E$ is a Helson set, and if $s \in P_{E}(G)$, then no point of $U \cap E$ can belong to the singular support of $s$.

In proving this we may (since the problem is local) assume that $E$ is compact. It then suffices to show that if $V$ is an open set meeting $E$ and such that $\nabla \subset U$, then no point $x_{0} \in V \cap E$ belongs to sing supp s. However the open sets $U$ and $\nabla^{\prime}$ cover $G$ and elements $u$ and $v$ of $A(G)$ may be chosen so that supp $u \subset U$, supp $v \subset \nabla^{\prime}$, and $u+v=1$ on a neighbourhood of $E$. Then $s=u s+v s$. Here

$$
\begin{aligned}
& \text { supp } u s \subset \operatorname{supp} u \cap \operatorname{supp} s \subset \bar{U} \cap E \\
& \text { supp } v s \subset \operatorname{supp} v \subset V^{\prime} .
\end{aligned}
$$

Since $\bar{U} \cap E$ is a Helson set, $u s$ is a measure (Lemma (3.1)); and in any case $v s=0$ on a neighbourhood of $x_{0}$. Thus $s$ is equal on a neighbourhood of $x_{0}$ to a measure.

(2.3.2) Corollary. The conclusion of Corollary (2.2.4) holds whenever $E \subset F$ are closed subsets of $G$ and $E$ satisfies condition $(N H)$ of Theorem (2.3).

Proof. Take $s_{1} \in P_{E}^{0}(G) \subset P_{E}(G)$ such that sing supp $s_{1}=E$, and let $\lambda$ be as in the proof of Corollary (2.2.4). Then $s=s_{1}+\lambda$ again satisfies all the demands.

\section{A lemma and its proof}

The lemma used in the proofs of Theorem (2.3) and Remark (2.3.1) has its own interest. It is almost certainly known, but I know of no suitable reference. 
(3.1) LeMMA. Let $H$ be a closed subset of $G$. The following statements are equivalent:

(i) $H$ is a Helson set.

(ii) $P_{H}^{0}(G) \subset M(G)$.

Note. We diverge from Rudin's convention ([2], p. 114) by not insisting that a Helson set be compact; we ask only that it be closed. Provided $C(H)$, the space of continuous functions on $H$, is replaced by $C_{0}(H)$, the subspace formed of those elements of $C(H)$ which tend to zero at infinity on $H$, this change of footing involves no essential changes.

Proof. Define

$$
A_{H}(G)=\{u \mid H: u \in A(G)\},
$$

where $u \mid H$ signifies the restriction of $u$ to $H ; A_{H}(G)$ is thus a subspace of $C_{0}(H)$. We norm $A_{H}(G)$ by setting

for $f \in A_{H}(G)$.

$$
\|f\|=\inf \left\{\|u\|_{A}: u \in A(G), u \mid H=f\right\}
$$

We shall consider the injection map $T$ of $A_{H}(G)$ into $C_{0}(H)$. It is clear that $T$ is continuous. The Weierstrass-Stone theorem shows that the range of $T$ is dense. Statement (i), which evidently means that $T$ is onto, is thus equivalent to the statement that $T$ has a continuous inverse.

On the other hand it is easily verified that the dual of $A_{H}(G)$ can be identified with $P_{H}^{0}(G)$ in such a way that the adjoint $T^{\prime}$ of $T$ carries each bounded Radon measure on $H$ (regarded as a linear functional on $C_{0}(H)$ ) into the pseudomeasure on $G$ which it defines, this pseudomaesure being automatically a member of $P_{H}^{0}(G)$. (In this connection it should be remembered that a bounded Radon measure on $H$ may be identified with an element of $M(G)$ whose support is contained in $H$.) Accordingly, statement (ii) signifies that $T^{\prime}$ is onto.

Finally, a well-known theorem (see, for example, [3], Corollary 8.6.15) says that $T$ has a continuous inverse if and only if $T^{\prime}$ is onto. Whence the equivalence of (i) and (ii).

Remark. Save for the case in which $H$ is known to be a spectral synthesis set, it is an interesting open problem to know whether one may in (3.1.ii) replace $P_{H}^{0}(G)$ by $P_{H}(G)$ and still obtain a statement equivalent to (3.1.i). However, we do have the following (known) corollary.

(3.2) Corollary. If a closed set $H \subset G$ is such that $P_{H}(G) \subset M(G)$ (i.e., if $H$ supports no true pseudomeasures), then $H$ is a Helson set.

Proof. This is an immediate consequence of (3.1) and the trivial inclusion $P_{H}^{0}(G) \subset P_{H}(G)$. 


\section{References}

[1] Kahane, J.-P., et Salem, R. Ensembles parfaits et séries trigonométriques. Actualités Sci. et Ind. 1301. Paris (1963).

[2] Rudin, W. Fourier analysis on groups. Interscience Publishers (1962).

[3] Edwards, R. E. Functional A nalysis: Theory and A pplications. Holt, Rinehart and Winston Inc., New York (1965).

[4] Pollard, H. The harmonic analysis of bounded functions. Duke Math. J. 20 (1953), 499512.

[5] Gaudry, G. I. Multipliers of type $(p, q)$. To appear, Pacific J. Math.

[6] Edwards, R. E. Spans of translates in $L^{(P}(G)$. J. Australian Math. Soc. 5 (1965), $216-233$.

[7] Edwards, R. E. Uniform approximation on noncompact spaces. To appear, Trans. Amer. Math. Soc.

Department of Mathematics

Institute of Advanced Studies

Australian National University 